\title{
Transformaciones urbanas como estrategia de desarrollo urbano sostenible: experiencias, propuesta tipológica, prospectiva para Colombia*
}

\section{Urban transformations as a sustainable urban development strategy: experiences, typological proposal, prospective for Colombia}

\author{
Julián Rodrigo Quintero-González , (1) https://orcid.org/0000-0001-5238-4067, \\ Laura Camila Castro-Cubides****, (1) https://orcid.org/0000-0002-5066-0889 y \\ Adriana Yisel Soler-Bermejo****; , (I) https://orcid.org/0000-0002-7208-3648
}

Citar este artículo como: Quintero-González, J. R., Castro-Cubides, L. C. y Soler-Bermejo, A. Y.(2021). Transformaciones urbanas como estrategia de desarrollo urbano sostenible: experiencias, propuesta tipológica, prospectiva para Colombia. Revista Nodo, 15(30), pp. 45-60.

\section{Resumen}

Este artículo expone la naturaleza de las transformaciones urbanas como un modelo de planeación y gestión orientado a la recuperación y renovación de lugares y espacios en áreas urbanas y periféricas; su objetivo es identificar los beneficios en las dimensiones ambiental, social y económica de las ciudades y que se generan con la implementación de las transformaciones urbanas. La metodología empleada se orienta al estudio de experiencias exitosas en el contexto global, latinoamericano y colombiano, a través de la caracterización de los antecedentes y problemáticas en cada caso, los procesos de planeación y gestión, y los resultados de la implementación en torno al sistema medioambiental, físico, social y económico, que conforman una ciudad. En los resultados se presenta una propuesta tipológica de categorías de transformaciones urbanas de acuerdo con su naturaleza y los sistemas de ciudad sobre los cuales tienen mayores efectos; asimismo una discusión sobre su implementación en Colombia. Se concluye sobre la necesidad de una clasificación tipológica amplia

Fecha de recepción: 25 de julio de 2020 - Fecha de revisión: 15 de octubre de 2020

Fecha de aceptación: 30 de enero de 2021 • Fecha de publicación: 9 de abril de 2021

\footnotetext{
* Este artículo presenta el resultado del trabajo de investigación realizado por los autores en el marco del Grupo de Estudios e Investigación en Recursos Ambientales y Urbanos (RAU) perteneciente a la empresa AHCTTUM Quintero-G Ingeniería S.A.S., Colombia; el trabajo se encuentra encuadrado en las líneas de investigación de "Ambiente y Hábitat" y "Ciudad y Territorio" del Grupo RAU, y está orientado al estudio y análisis de la arquitectura e infraestructuras verdes, su naturaleza y potencialidades de desarrollo.

\%* Ingeniero en Transporte y vías. Magíster en Ingeniería Ambiental, UPTC. Magíster en Derecho Privado, Persona y Sociedad con Énfasis en Derecho del Transporte, Logística e Infraestructura. Director Ejecutivo y de Proyectos e Investigador del Grupo RAU de AHCTTUM Quintero-G Ingeniería S.A.S, Tunja, Colombia. Correo electrónico: jrquinterog.itv@gmail.com

*** Ingeniera en Transporte y Vías. Investigadora del Grupo RAU de AHCTTUM Quintero-G Ingeniería S.A.S., Tunja, Colombia. Correo electrónico: lalis02.s3@gmail.com

**** Ingeniera en Transporte y Vías. Investigadora del Grupo RAU de AHCTTUM Quintero-G Ingeniería S.A.S., Tunja, Colombia. Correo electrónico: adrianasoler386@gmail.com
} 
y aplicable en diversos contextos, y sobre el escenario de implementación de las transformaciones urbanas en las ciudades colombianas.

Palabras clave: Zona urbana, Obras públicas, Calidad de vida, Planificación urbana, Desarrollo sostenible.

\section{Abstract}

This article exposes the nature of urban transformations as a model of urban planning and management-oriented towards the recovery and renovation of places and spaces in urban and peripheral areas. The objective is to identify the benefits in the environmental, social and economic dimensions of cities, and that are generated with the implementation of urban transformations. The methodology is oriented to the study of successful experiences in the global, Latin American and Colombian context; through the characterization of the antecedents and problems in each case, the planning and management processes, and the results of the implementation around the environmental, physical, social and economic system that make up a city. The results are a typological proposal of categories of urban transformations according to their nature and the city-systems on which they have the greatest effects; also a discussion on its implementation in Colombia. It concludes on the need for a broad typological classification applicable in various contexts, and the implementation scenario of urban transformations in Colombian cities.

Keywords: Urban area, Public works, Quality of life, Urban planning, Sustainable development.

\section{Introducción}

Las problemáticas socio-ambientales urbanas que se extienden a todos los sistemas que conforman a las ciudades -el sistema físico, el sistema medioambiental, el sistema social y el sistema económico (Velásquez, 2012)-, están represen- tadas principalmente por la ausencia de espacios públicos y áreas verdes, la contaminación del aire, los problemas de saneamiento, la congestión de tráfico, la baja calidad de vida urbana, la pérdida de identidad urbana y la limitada dinámica económica, entre otras. Estas problemáticas han sido tratadas a través de las denominadas transformaciones urbanas, como por ejemplo la restauración y/o recuperación de espacios naturales deteriorados y la revitalización y transformación de infraestructuras públicas (Diputación de Barcelona, 2008). La realización de este tipo de intervenciones en las ciudades ha tomado bastante fuerza dentro de los modelos de planeación y diseño de estrategias urbanas, dado que su implementación ha traído consigo beneficios en las dimensiones social, ambiental, económica y cultural, relacionadas con la sostenibilidad urbana. Es así como se encuentran experiencias principalmente en las ciudades de las regiones de Europa y Asia, en donde la conceptualización de la transformación urbana, su entendimiento como herramienta práctica en los procesos de mejora de la calidad de vida urbana, y el conocimiento decantado durante años de experiencia en la implementación de este tipo de intervenciones, han resultado en una amplia gama de propuestas innovadoras desarrolladas por parte de arquitectos e ingenieros; en la mayoría de los casos han sido exitosas y han provisto soluciones adecuadas a las problemáticas urbanas.

En el contexto de la región latinoamericana la incursión de las transformaciones urbanas es aún tímida, a la vez que se empiezan a dar las primeras experiencias exitosas en las ciudades de los países más grandes y con mayor conocimiento e investigación en la materia; en el caso de Colombia este conocimiento aún es escaso y existen inquietudes acerca de lo que encierra el concepto de transformación urbana. Sobre la base de lo anterior, si bien en los países más desarrollados ya se cuenta con conceptualizaciones claras acerca de lo que es una transformación urbana, y podrían tomarse como referentes para ser aplicadas en el planteamiento de proyectos urbanísticos en las ciudades latinoamericanas y colombianas, se hace necesario 
realizar una reflexión acerca de los conceptos más representativos logrados hasta el momento, así como esbozar una distinción entre las diferentes tipologías derivadas de las características particulares de los proyectos más destacados hasta ahora desarrollados.

El presente artículo tiene como objeto conceptualizar la transformación urbana; exponer las experiencias más sobresalientes en el ámbito global, latinoamericano y colombiano; y a partir de lo anterior, formular una propuesta de familias o clases de transformaciones urbanas que sirva como base para su identificación y entendimiento por parte de profesionales, expertos y administradores, como punto de partida para su formulación, gestión y diseño, en los procesos de planeación urbana.

\section{Conceptualización de la transformación urbana}

De manera general no se identifica una definición unívoca del término transformación urbana, siendo más bien un concepto amplio resultado de las teorías de la planificación urbana y los procesos urbanos desarrollados en las ciudades. Es precisamente este pensamiento el que postulan Zeren y Gürler (2011), quienes argumentan que la definición del concepto de transformación urbana en la teoría de la planificación cambia en cada período, al mismo tiempo que el enfoque de la transformación urbana en la práctica de la planificación difiere con respecto a los cambios de paradigma en la historia de la planificación lo que conlleva a un entendimiento temporal distinto del término transformación urbana, en consonancia con cada nueva manifestación de procesos urbanos, estos últimos sujetos a una evolución que puede extenderse de momentos históricos puntuales hasta siglos enteros. No obstante lo anterior, estos autores ofrecen una aproximación moderna al concepto, al señalar que los enfoques para la transformación urbana se centran en la regeneración urbana integrada que resulta del desarrollo de modelos estratégicos tanto a nivel organizativo como espacial. El propósito de la transformación urbana puede ser definido conforme a lo expuesto por Mishra y Pandit (2013) quienes indican que su objetivo es mejorar la calidad social, económica y espacial de las ciudades.

De otra parte, sobre la base de la exposición de ejemplos gráficos de transformaciones urbanas en el marco global y en el contexto colombiano, así como de la revisión de diversos casos de proyectos efectuados en dichos ámbitos, derivados del trabajo de investigación realizado por Quintero y Quintero (2018), pueden identificarse manifestaciones puntuales de las transformaciones urbanas, a saber: la conservación del patrimonio histórico, arquitectónico, cultural y antropológico; la transformación de áreas urbanas degradadas; la renovación de espacios urbanos deteriorados; la recuperación y generación de espacio público; la revitalización de las corrientes de ríos y canales urbanos; la construcción de parques lineales; la consolidación de ejes ambientales; la utilización de infraestructuras de arquitectura verde viva; y de manera particular la restauración de áreas industriales abandonadas, y la implementación de sistemas de transporte limpios.

\section{Experiencias exitosas}

\section{Marco global}

En Asia se cuenta con casos exitosos que bien pueden considerarse hitos en lo relativo al desarrollo de proyectos de transformación urbana, y de manera general de la implementación de una adecuada planeación y gestión de ciudades. En este contexto puede mencionarse al proyecto de transformación urbana del río Cheonggyecheon, que de acuerdo con Development Asia (2016) fue un curso de agua que atravesaba el centro de Seúl en Corea del Sur, el cual se vio drásticamente afectado por la rápida urbanización que se desarrolló a mediados del siglo XX; en la década de 1940 la orilla del río se encontraba abarrotada por viviendas parecidas a chozas, construidas a partir de materiales de baja calidad, y en donde además el saneamiento básico 
se convirtió en un problema. Para resolver esta situación el río se cubrió con concreto y se convirtió en una carretera de $6 \mathrm{~km}$ a fines de la década de 1950. En 1971 Cheonggyecheon tenía una autopista elevada de $5,8 \mathrm{~km}$ y 6 carriles para acomodar el creciente tráfico vehicular en la capital. Con el pasar de los años se hicieron visibles las problemáticas de salubridad e incluso de índole estructural en la autopista construida, cuyo tratamiento, al ser analizado por la Sociedad Coreana de Ingeniería Civil, supondría un muy alto costo; es así que por decisión del Gobierno Metropolitano de Seúl, en cabeza del entonces Alcalde Lee Myung-bak se toma la decisión de desarrollar el proyecto de revitalización de la corriente de Cheonggyecheon.

Como derroteros del proyecto se consideraron el retiro de la autopista y la restauración del río, la consolidación de un corredor verde, el fortalecimiento de los sistemas de transporte público, y la integración urbana sostenible en el diseño de la infraestructura; estos aspectos dieron forma al proceso de renovación urbana. Como resultado se logró la restauración histórica, cultural y ambiental del Cheonggyecheon (Figura 1) (Kontentreal LLC, 2008). Según lo indica Development Asia (2016) el proyecto tardó 27 meses en desarrollarse, su apertura se realizó en el año 2005; el corredor ambiental cuenta con una longitud de $5,4 \mathrm{~km}$, un ancho máximo de $72 \mathrm{~m}$, y 22 puentes nuevos sobre el arroyo (5 para peatones y 17 para vehículos); se reintrodujeron docenas de nuevas especies, incluyendo aves y peces; se retiraron alrededor de 170.000 vehículos de la zona, y se redujo el $35 \%$ de partículas suspendidas; se redujo la temperatura en el área en $5{ }^{\circ} \mathrm{C}$; se logró un aumento de entre el $6 \%$ y $10 \%$ en el número de pasajeros en autobús durante el periodo de construcción, y de entre el 6 $\%$ y $10 \%$ en el número de pasajeros del metro en el mismo periodo; y el sitio es visitado por cerca de 64.000 personas diariamente y 23,4 millones al año. En síntesis, este proyecto se constituye en un referente mundial para el entendimiento de la concepción de gestión de ciudad y transformación urbana.

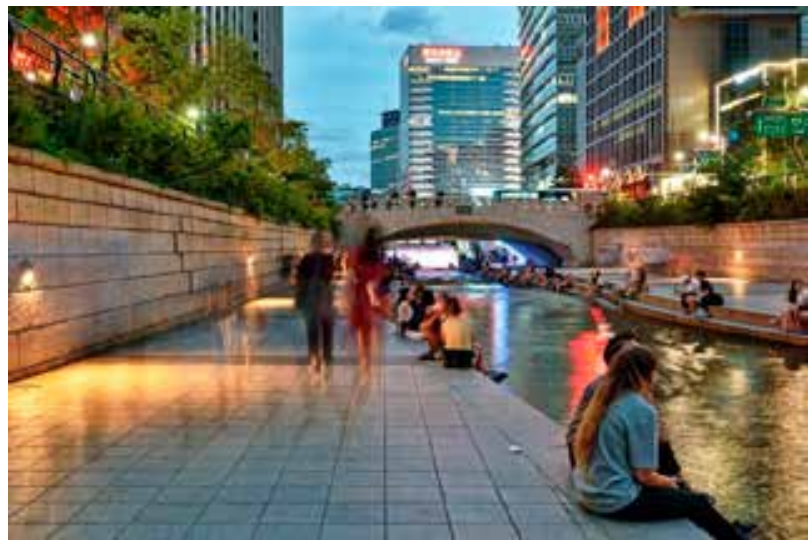

Figura 1. Resultado de transformación urbana río Cheonggyecheon. Fuente: James Kim, Pixabay.

Otro caso ejemplar es el del Tianjin Qiaoyuan Wetland Park, un parque de 22 hectáreas localizado en el Barrio de Hedong en Tianjin, China. En este lugar la rápida urbanización convirtió un campo de tiro periférico en un vertedero de basura y un sumidero de drenaje para aguas pluviales urbanas; el sitio estaba muy contaminado, sembrado de basura, desértico y rodeado de barrios marginales y estructuras temporales desvencijadas; de ahí surge la solicitud de los residentes de una mejora ambiental (ASLA, 2010). El Gobierno Municipal de Tianjin responde con la contratación del Arquitecto Paisajista Kongjian Yu y su equipo de Turenscape, expertos en diseño arquitectónico, de paisaje, urbano y ambiental (Green Planet Architects, 2018a). Sobre lo señalado por Yu (2013), el objetivo del equipo de diseñadores fue crear un parque que proporcionara una diversidad de servicios de la naturaleza para la ciudad y los residentes urbanos circundantes, incluida la contención y purificación de las aguas pluviales urbanas, la mejora del suelo salino-alcalino a través de procesos naturales, la oferta de medios para la educación ambiental y la recuperación del paisaje de manera estética.

El equipo diseñó "paletas de adaptación", haciendo referencia a estanques diseñados para permitir la adaptación de la naturaleza nativa (Green Planet Architects, 2018b); se pensó así en una estrategia de diseño regenerativo de paisaje simple, según la American Society of Landscape Architects (ASLA, 
2010), que incluyó la realización de 21 cavidades de estanque de 10 a 40 metros de diámetro y de uno a cinco metros de profundidad, creando así hábitats diversos en los que iniciaron los procesos naturales de adaptación de las plantas. El Tianjin Qiaoyuan Wetland Park, cuya construcción tardó dos años y fue entregado al servicio en 2005, ha traído consigo diversos beneficios ambientales (mejor alcalinidad, aumento de valor del hábitat en el sitio, captura de 539 toneladas de carbono), sociales (reducción de ruido, acceso a espacio verde, oportunidades educativas, conciencia ecológica), y económicos (ahorro en construcción), lo que ha redundado en una mayor accesibilidad a infraestructuras públicas y recursos naturales para los 20.000 residentes cercanos, siendo visitado por 350.000 personas cada año (Rottle y Lacson, 2011).

En Oceanía, particularmente en una zona de la ciudad de Christchurch en Nueva Zelanda, se encuentra el Pabellón de pallets; allí se realizó la transformación de un espacio público a principios de diciembre del año 2012 cuya idea más representativa fue el conjunto de materiales que se emplearon para adecuación del lugar y que le apuestan a la innovación por medio de la sostenibilidad ambiental; en este caso se implementaron pallets que son elementos generalmente fabricados en madera o plástico, usados como base para transportar mercancías de manera cómoda y segura (Icecomex, 2007). Según Martínez (2013) debido a la carencia de centros culturales y teatros, derrumbados por episodios de terremotos que han ocurrido en Christchurch, la organización Gap Filler -grupo neozelandés dedicado a la regeneración de espacios- se dio a la tarea de desarrollar el proyecto, que además contempló la reconexión de la calle Victoria con la plaza homónima.

$\mathrm{Su}$ gran acogida social se da principalmente por su estilo diferente y creativo. Tomando como base lo expuesto por Martínez (2013), al principio el Pabellón de pallets fue un proyecto que duraría seis meses en funcionamiento; pero debido al éxito que tuvo con la comunidad se realizaron diferentes donaciones para oficializar el lugar de forma definitiva, con lo cual no solo se logró mantener la estadía del centro cultural, sino que se mejoraron las instalaciones, la seguridad y se ofrecieron nuevos servicios, demostrando ser un caso ejemplar de transformación urbana por el gran acogimiento social que ha tenido desde sus inicios.

En Europa se pueden encontrar manifestaciones de transformaciones urbanas interesantes dada su particularidad, pero también su posible amplia aplicabilidad. Este es el caso del Duisburg Nord Landscape Park, en Alemania. Conforme lo relata Stilgenbauer (2005), el parque se encuentra localizado en el corazón del densamente poblado distrito alemán de Ruhr, que hasta inicios de 1970 se constituía en uno de los centros de fabricación de acero y minería de carbón más grandes de Europa; luego aquellas industrias tuvieron un declive que precipitó una crisis económica y ecológica acompañada de un cambio social y una pérdida de identidad cultural en la región; a la postre sólo quedó un paisaje extraño de camas de ferrocarril, chimeneas, montones de escoria, suelos contaminados, ruinas industriales y vías navegables rediseñadas. Stilgenbauer (2005) menciona que la autoridad de desarrollo territorial de North Rhine-Westphalia, apoyada por un fondo de bienes raíces, compró los terrenos de la antigua planta de fabricación de Thyssen Steelworks; al mismo tiempo la ciudad de Duisburg cambió su zonificación para permitir su conversión en un parque público; esto abrió las posibilidades para que el diseñador paisajista Peter Latz desarrollara su idea de transformación urbana.

El paisaje postindustrial, denominado así por el grupo de Latz, consiste según su entendimiento en la consolidación de un diseño paisajístico a través de elementos aceptados y perturbadores, tanto armónicos como interrumpidos, obteniendo como resultado una metamorfosis del paisaje sin destruir las características existentes (Latz und Partner, s.f.). Esta filosofía permite lograr el resultado descrito por Stilgenbauer (2005), quien enuncia que el proyecto del Duisburg Nord Landscape Park cuenta con 230 hectáreas; se desarrolló en fases 
a lo largo de trece años, desde 1989 hasta 2002; está compuesto por varias capas de elementos de diseño que funcionan de manera independiente como sistemas de parques y ayudan a crear un sentido de orientación; esto permite hacer de un lugar extremadamente complejo algo posible de interpretar a escala humana. Además se recuperó el río Emscher que atraviesa el parque desde el este al oeste, restableciendo su funcionamiento ecológico como hábitat para plantas acuáticas y la vida silvestre. En resumen, el parque que atrae a más de 500.000 visitantes cada año, conforme lo alude su sitio oficial Landschaftspark Duisburg-Nord (s.f.), ofrece jardines, prados, cuerpos de agua y una naturaleza muy desarrollada, que recupera su territorio de la industria (Figura 2).

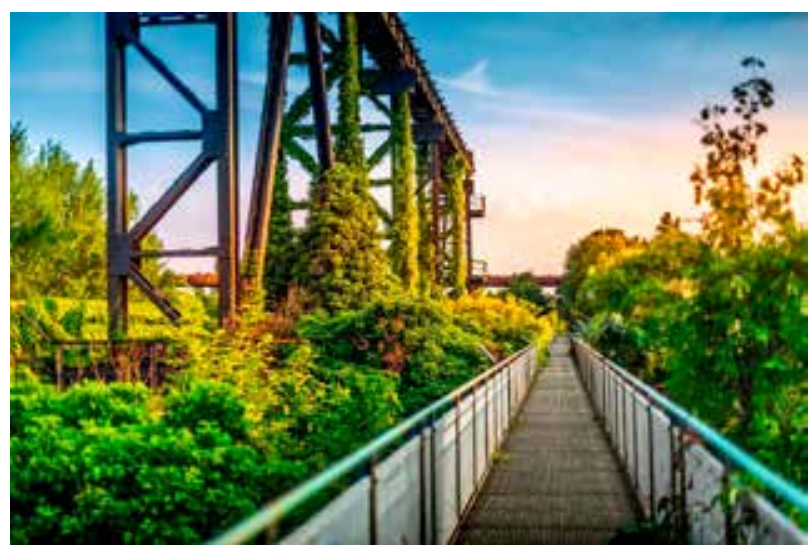

Figura 2. Instalaciones regeneradas, hoy Landschafts Park Duisburg-Nord. Fuente: https://pixabay.com/es/ photos/landschaftspark-duisburg-nord-3463279/

En Estados Unidos, específicamente en New York, el High Line Elevated Park se encuentra dentro de los casos más representativos de regeneración de espacios urbanos; la historia inicia entre 1929y 1934 con la construcción de una línea de tren elevada a unos 10 metros de altura de las principales vías de Manhattan, para evitar los peligros que existían en las mismas, donde el tránsito se veía expuesto a peligros por la circulación de trenes junto con los demás vehículos. La línea de tren funcionó aproximadamente 50 años, luego de quedó en completo abandono. Así, en 1999 se pretendió demoler la estructura; pero esto no se logró debido a un grupo defensor - Amigos de la High Line- que propuso recuperar el espacio dando un enfoque medioambiental, cultural y comercial (Lindner y Rosa, 2016). Gracias a esto se logró transformar la estructura en un atractivo recorrido peatonal elevado de más de 2,0 kilómetros de longitud, el cual funciona con un pavimento que posibilita el crecimiento de plantas; esta transformación obedece a la idea de agritectura (combinación de agricultura con arquitectura) (Ciudades del Futuro, 2012). Según Sánchez (2017), este parque está conformado

por caminos, zonas de descanso, jardines florales, teatro, con butacas lineales de madera escalonada que finalizan en un gran ventanal que nos permite ver la circulación de autos dentro de la ciudad, sin estar en ella. [...] Dividido en tres secciones, se puede acceder por diversos puntos de la ciudad. Tiene nueve accesos en total, cuatro de ellos con ascensor. Cuenta con escaleras y rampas para sillas de ruedas (párr. 3-6).

La popularidad de esta transformación se evidenció en las cifras registradas de las visitas hechas a dicho parque, que según Hammond (2011) en 2010 alcanzaron los 2 millones de visitas, y por consiguiente se ha originado gran desarrollo económico y arquitectónico en la ciudad.

En Toronto, Canadá, se aprecia un caso representativo de la recuperación y aprovechamiento de una zona industrial antigua; se trata del Distillery District, sus instalaciones se ubicaron en pleno centro de la ciudad. Según un artículo publicado por Culture Trip (2016), en 1871 logró convertirse en una potencia en la industria del alcohol, originando gran cantidad de exportaciones alrededor del mundo; por consiguiente generó un gran aporte a la economía británica. Según Marván (2018) en 1990 la destilería dejó de producir, pero por sus características de diseño, el lugar se prestó para filmar algunas películas y trece años después de su declive la destilería fue comprada por Cityscape Holdings Inc., quienes vieron en esta zona un espacio perfecto para representar la cultura europea, esta transformación lo convirtió 
en un lugar histórico. Conforme a Donat (2015) en aproximadamente $32.000 \mathrm{~m}^{2}$ y 40 edificios, se representa la combinación de entretenimiento, creatividad e historia en un diseño bastante contemporáneo, donde se conservan diseños industriales que predominaban en la época victoriana. En la actualidad se ha convertido en un sitio turístico de Toronto destinado a promover el arte, la cultura y el ocio; en él se encuentran varias tiendas, cafés, restaurantes, galerías de arte, entre otras empresas productivas (Figura 3); además se realizan eventos culturales de todo tipo, lo cual atrae un gran número de visitantes por el peculiar ambiente que predomina en esta zona.

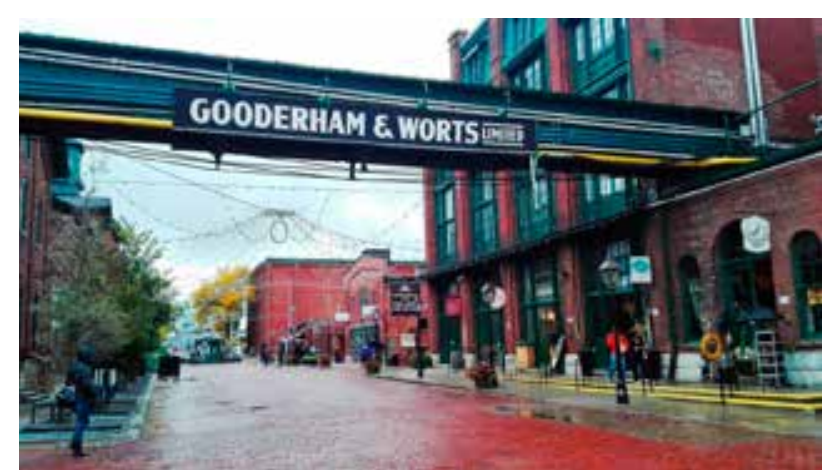

Figura 3. Restauración y reutilización de las edificaciones. Fuente: https://pixabay.com/es/photos/toronto-canad \%C3\%A1-arquitectura-2303875/

\section{Contexto latinoamericano y colombiano}

Una de las ciudades pioneras en estas transformaciones es Curitiba, capital del Estado de Paraná en Brasil y que para el año 2017 contaba con una población aproximada de 1.950 .000 habitantes (Pascuali, 2020). Según lo indica Fukuda (2010), debido al crecimiento que se fue dando a través de los años, se inicia un proceso para conservar el medio ambiente por medio de acciones para prevenir inundaciones y promover la conservación de los fondos de los valles y zonas verdes. Para el ordenamiento de la ciudad se implementaron leyes que reglamentan los procesos de transformación y la planificación urbana; por ello existe el Instituto de Investigación y Planificación Urbana de Curitiba (IPPUC), encargado de manejar y controlar las inversiones sociales y ambientales, la preservación de las condiciones ambientales y el mantenimiento de zonas verdes, logrando resultados exitosos de sostenibilidad ambiental en la ciudad. Según Fukuda (2010)

Como resultado de las acciones de la ciudad de Curitiba, hay 33 áreas de conservación, con un total de $18^{\prime} 400.000 \mathrm{~m}^{2}$ de áreas verdes y bosques, $120 \mathrm{~km}$ de carriles de bicicleta que conectan los parques, 300 mil árboles en la vía pública que están geo-referenciados para que pueda hacerse un programa más adecuado de la poda y mantenimiento (p. 67).

Se aprecia la participación significativa de las zonas verdes dentro de la ciudad (Figura 4), además se han adaptado políticas públicas como la prohibición estacionamiento en algunos lugares para reducir la emisión de gases de los vehículos y disminuir la contaminación haciendo que las lluvias no causen inundaciones. Según un artículo publicado por Paisajismo Digital (2017) la transformación de Curitiba ha logrado sincronizar una eficiente red integrada de transporte, con gran capacidad de pasajeros, parques, jardines, zonas verdes, planes de recolección de basuras y demás sistemas que componen la ciudad e intervienen en el buen funcionamiento y calidad de vida para sus habitantes.

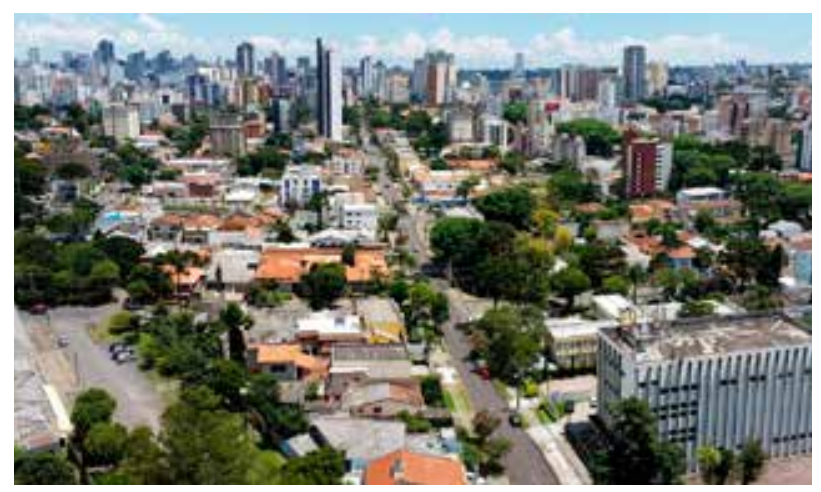

Figura 4. Panorámica Centro de Curitiba. Fuente: https://pixabay.com/es/photos/curitiba-centro-hori zonte-calidad-2778943/ 
En Colombia se descubren varios casos interesantes a examinar; un referente ejemplar de la recuperación del patrimonio histórico y cultural se encuentra en la antigua Plaza de Mercado de la ciudad de Tunja, hoy Centro Cívico y Comercial Plaza Real. De acuerdo con la actual Administración hacia el año 1913 mediante la Ley 8, el Gobierno Nacional toma la iniciativa de efectuar la conmemoración del primer centenario de la Independencia de Colombia, para lo cual promueve la realización de la Exposición Nacional de Ciencias, Artes, e Industrias; para tal fin ordena la construcción de los pabellones requeridos para el evento, y que posteriormente pudieran ser utilizados como plaza de mercado. En 1919 se terminó el pabellón central, entre 1923 y 1939 se adicionaron los pabellones laterales, con lo que se celebró el Cuarto Centenario de la Fundación de la ciudad de Tunja; se destaca del diseño las fachadas neoclásicas, la amplia cúpula de influencia francesa, y las columnas con capiteles corintios; en el año 1976 pasó de ser propiedad del municipio a la Empresa de Obras Sanitarias de Tunja, y de ésta al Instituto de Beneficencia y Lotería de Boyacá en 1979, que el 1990 entregó el inmueble para su restauración, siguiendo lo establecido en la Resolución 12 de 1982 del Consejo de Monumentos Nacionales, que en aquel año lo declaró como Monumento Nacional.

Señala la actual Administración que los trabajos de reconstrucción inician con los pabellones laterales en el año 1993, siendo terminados en noviembre de 1994; de la misma manera, en 1996 se inician los trabajos en el pabellón central, siendo terminados en diciembre de 1997; los pabellones laterales albergan 38 locales y 26 oficinas, al mismo tiempo que el pabellón central aloja 34 locales y 53 oficinas; el grupo de diseñadores fue constituido por Rafael Obregón, Edgar Bueno y Orlando Ruiz, de Obregón Bueno y Cia., la gestión de gerencia y construcción fue realizada por la firma C.M.S. Arquitectos, dirigida por el Arquitecto Camilo Manrique Santa María y la Organización de Promociones Inmobiliarias. Hoy por hoy, el Centro Cívico y Comercial Plaza Real representa un ejemplo de transformación urbana física y funcional, proporcionando diversos servicios comerciales, de negocios y culturales a la ciudad; cuenta con 72 locales, 79 oficinas, ascensores, áreas de administración, un museo, y amplios jardines y espacio público (Figura 5); se constituye así en hito de la planeación y transformación urbana de la ciudad, así como un monumento que promueve la identidad urbana y apropiación.

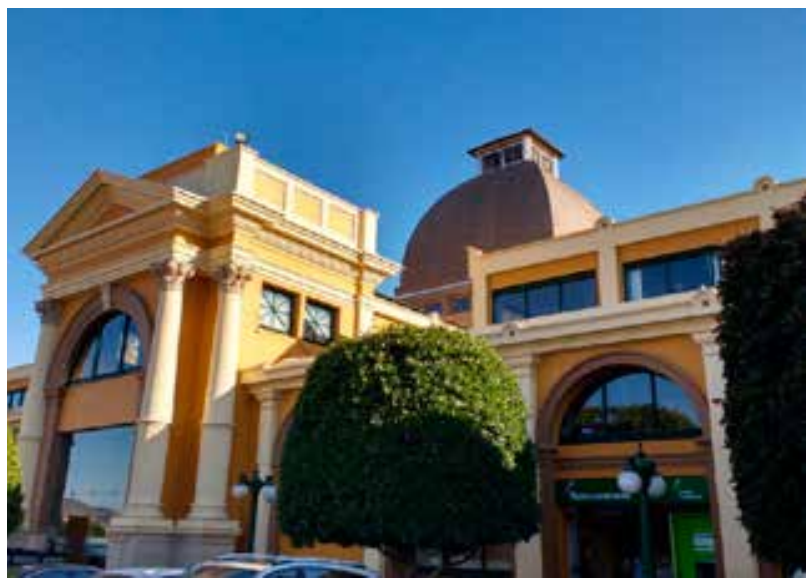

Figura 5. Centro Cívico y Comercial Plaza Real, Tunja. Fuente: (c) Julián Rodrigo Quintero González (fecha de captura: 2018/03/12)

Otro caso a considerar es el Eje Ambiental sobre la Avenida Jiménez en la ciudad de Bogotá D.C.; según Motoa (2018) la Avenida Jiménez ubicada en el centro de la ciudad -y que en el año 2018 cumplió 80 años-, tuvo sus inicios como un cauce contaminado -llamado Río San Francisco-, por el cual corrían aguas negras y desechos químicos e industriales; esta situación afectaba la salud pública, lo que provocó que el Gobierno Nacional ordenara su canalización mediante la Ley 10 de 1915. Añade Motoa que el Concejo de Bogotá mediante el Acuerdo 31 de 1917 le dio el nombre de Avenida Jiménez de Quesada, la cual tendría su primer tramo terminado en el año de 1938; luego de este periodo y hasta la década del 90 la vía fue utilizada por un sin fin de peatones y vehículos, estos últimos en un exceso que llevó al colapso de la vía; se decide entonces, en el año 2000, la intervención del corredor a través de la renovación 
urbana y específicamente la consolidación del hoy conocido Eje Ambiental (Figura 6).

Esta transformación brindó beneficios ambientales, sociales y económicos en torno a la intervención urbanística realizada, e incluso llegó a convertirse en un ícono para la ciudad y ejemplo para el país en temas de transformación urbana; según lo describe Lozano (2016), desde lo jurídico esto fue posible gracias a un completo cambio de uso del suelo, pasando de una vía congestionada a un espacio público diferenciado para peatones. Se identifica como fortaleza el planteamiento de las políticas públicas y de ciudad, al encontrar históricamente -para el caso en comento- instrumentos jurídicos que promovían y desarrollaban la renovación urbana del espacio público; el caso del Eje Ambiental sobre la Avenida Jiménez se constituye en un claro ejemplo de las bondades de la renovación urbana y del espacio público.

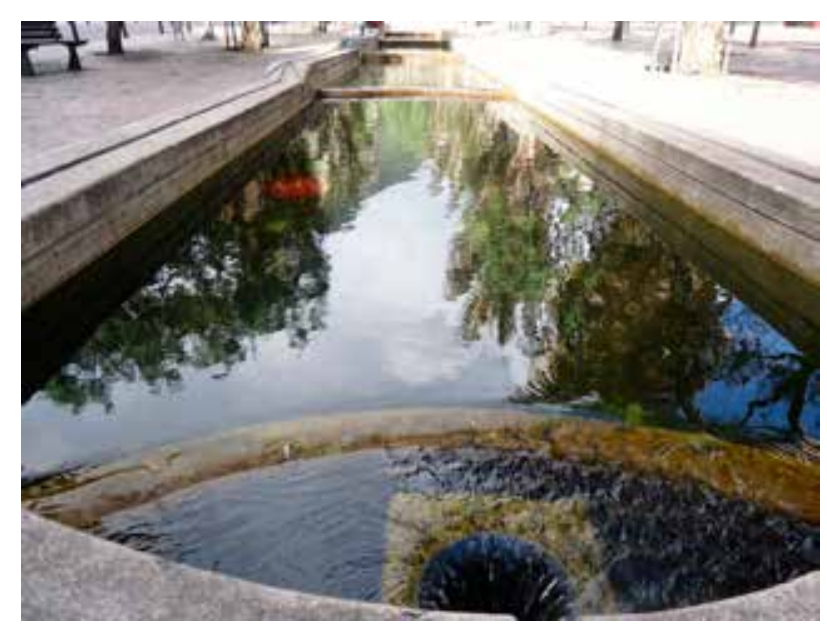

Figura 6. Canalización Río San Francisco, hoy Eje Ambiental Centro de Bogotá D.C. Fuente: https://pixabay.com/es/ photos/bogota-agua-cundinamarca-colombia-54427/

Medellín cuenta con el Tranvía de Ayacucho y sus dos cables. Históricamente existió un tranvía desde 1887 que primeramente fue de tracción animal, pero en 1919 en Medellín se implementó el tranvía eléctrico que operó hasta 1950 (EPM, 2014). Según Villegas (1995, citado por Márquez, 2012) el tranvía dejó de funcionar debido a "efectos económicos de alta competencia del transporte público automotor, cuya demanda crecía aceleradamente" (p. 145). Luego, para poder reinventarse y salir de la crisis se plantean las transformaciones urbanas como medio para mejorar las condiciones de vida de los habitantes; de allí se levantan grandes obras de infraestructura para la ciudad y por supuesto el tranvía inaugurado en el año 2016 (Figura 7). Para Linde (2017), la imagen paisajística del barrio Ayacucho antes de la construcción del tranvía era desolada y peligrosa; por tanto, se buscó la forma de erradicar este panorama dando espacio a la construcción de una de las estaciones que hoy en día "representa la posibilidad para más de $350 \mathrm{mil}$ personas de las comunas 8,9 y 10 de acceder de manera ágil, económica y limpia, a diversos lugares del área metropolitana" (Metro de Medellín, 2015, p. 5). Según Bueno (2016):

Con una longitud de $4,2 \mathrm{~km}$, moviliza a 90.000 pasajeros diariamente, desde la estación San Antonio de la Línea A del Metro, situada en la zona centro de la ciudad, hasta el barrio Alejandro Echavarría, ubicado en la Comuna 9 de la zona centro oriental de la misma. Su recorrido es de 12 minutos, con una frecuencia regular de cuatro, y beneficia a cerca de 350 mil personas de las comunas 8 Villa Hermosa, 9 Buenos Aires y $10 \mathrm{La}$ Candelaria de la zona centro oriental de la ciudad de Medellín. Tiene una velocidad promedio de $30 \mathrm{~km} / \mathrm{h}$ y se distribuye en seis paradas y tres estaciones de trasferencia. Se complementa con dos cables en su extremo oriental: uno entre Las Estancias y el 13 de noviembre; otro que recorre el tramo Villa Liliam-La Sierra (p. 98).

Con ello se evidenciaron las externalidades positivas derivadas de la construcción del tranvía. En la página oficial del Metro de Medellín (2016), se indica que uno de los aportes más importantes tiene que ver con la calidad del aire, gracias a que

el tranvía funciona con energía eléctrica, se dejarán de emitir 7.357 toneladas de $\mathrm{CO}_{2}$ a la atmósfera al año. [...] Los impactos positivos también se reflejarán en la salud de los habitantes de la zona. Se estima que gracias al tranvía se evitarán 23 casos de muertes 
asociadas a PM10 (material particulado). [...] Se calcula entonces que el uso del tranvía permitirá evitar 38 muertes por accidentalidad al año (párr. 16-18).

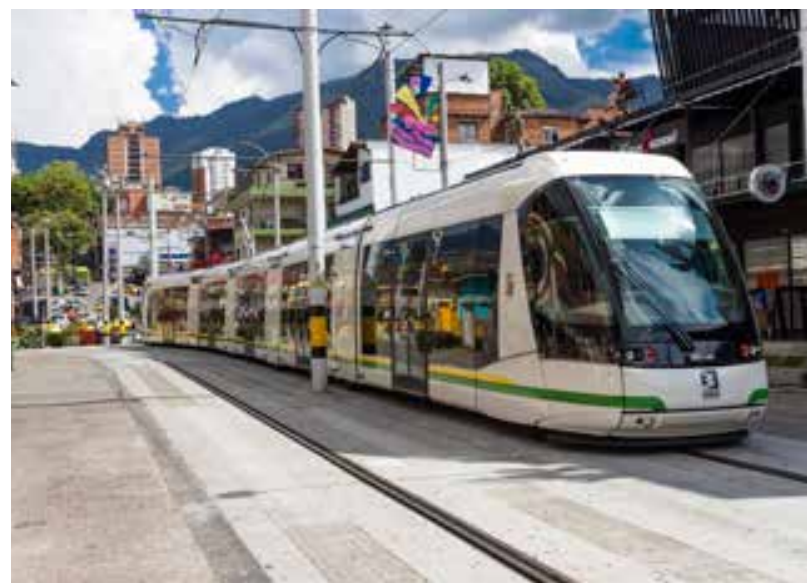

Figura 7. Tranvía de Ayacucho en funcionamiento actualmente. Fuente: https://pixabay.com/es/photos/calle-ciudadtr\% $\%$ C3\%A1fico-carretera-3105232/

\section{Propuesta tipológica}

Las clasificaciones disponibles en la literatura se encuentran marcadas por las experiencias y hallazgos en los estudios realizados en diferentes regiones, territorialidades, o áreas urbanas; aquellas clasificaciones han surgido como resultado de las múltiples estrategias de transformación urbana aplicadas como solución a las particulares problemáticas físicas, espaciales, sociales y ambientales en cada lugar. Pueden encontrarse clasificaciones convenientes y factiblemente aplicables; este es el caso de la clasificación expuesta por Zeren y Gürler (2011, p. 11), la cual distingue tres tipos, a saber: 1. Transformación urbana basada en la conservación del patrimonio en las áreas urbanas que tienen un significado histórico y cultural en la ciudad; 2. Transformación urbana basada en la regeneración de las áreas urbanas existentes con potencial económico y funcional; y 3 . La renovación urbana basada en el desarrollo de espacios urbanos deteriorados en la que estos se pueden modernizar y reestructurar. Una clasificación con un mayor grado de distinción es la propuesta por
Quintero y Quintero (2018) que la definen de la siguiente manera:

1) Transformación de conservación - orientada a la protección del patrimonio histórico, arquitectónico y urbanístico de la ciudad-; 2) Transformación de consolidación -aplicada a unidades morfológicas urbanas, espaciales, residenciales, industriales, comerciales, ambientales y de servicios en el área urbana y las periferias, mediante un enfoque hacia el desarrollo urbano sostenible de la ciudad como un todo-; 3) Transformación de renovación urbana -entendida como la regeneración urbana, reconstrucción, restauración, y/o revitalización de unidades morfológicas que han perdido sus cualidades espaciales y ambientales; como en el caso de las infraestructuras públicas y los recursos naturales-, y 4) Transformación de desarrollo, en la que se asume una orientación hacia la modernización urbana y la restauración y aprovechamiento socioeconómico (p. 81).

Las clasificaciones expuestas se consideran acertadas y al mismo tiempo aplicables a diversos contextos urbanos. No obstante lo anterior, el presente trabajo busca proponer una clasificación sobre la base de las diversas experiencias de transformaciones urbanas implementadas en un amplio espectro, y cuya naturaleza y características particulares permitan lograr una propuesta más profunda. Para esto se consideran como criterios en primer lugar, los sistemas que componen la ciudad, clasificados por Velásquez (2012) como: sistema económico, sistema social, sistema físico y sistema medioambiental; en segundo lugar, los ciclos del ecosistema urbano propuestos por Higueras (2013), en los cuales las transformaciones urbanas pueden tener influencia, aquellos son: el ciclo atmosférico, el ciclo hidrológico, el ciclo de materia orgánica y residuos, y el ciclo energético. El contraste de los anteriores criterios y los casos expuestos en el apartado anterior, relativos al ámbito global y latinoamericano así como el contexto colombiano, permiten logran la siguiente propuesta tipológica (Tabla 1.). 
Tabla 1. Propuesta tipológica de transformaciones urbanas

\begin{tabular}{|c|c|c|c|c|c|}
\hline Tipo & Descripción & $\begin{array}{l}\text { Casos } \\
\text { Representativos } \\
\text { Ciudad y país }\end{array}$ & Intervención & $\begin{array}{c}\text { Sistema de } \\
\text { Ciudad }\end{array}$ & $\begin{array}{c}\text { Modelo de } \\
\text { Financiamiento } \\
\text { Identificado }\end{array}$ \\
\hline \multirow{2}{*}{$\begin{array}{l}\text { Conservación } \\
\text { Ambiental }\end{array}$} & $\begin{array}{l}\text { Recuperación de } \\
\text { espacios naturales } \\
\text { que han sido alterados } \\
\text { por la acción del ser } \\
\text { humano, como invasión } \\
\text { con construcciones, } \\
\text { vertederos de basura, } \\
\text { uso de sustancias } \\
\text { contaminantes, entre } \\
\text { otras actividades que }\end{array}$ & $\begin{array}{l}\text { Tianjin Qiaoyuan } \\
\text { Wetland Park } \\
\text { (China) }\end{array}$ & Ambiental & Medioambiental & Fondos públicos \\
\hline & $\begin{array}{l}\text { con el paso de los } \\
\text { años han generado un } \\
\text { verdadero problema } \\
\text { ambiental. La } \\
\text { transformación de } \\
\text { conservación ambiental } \\
\text { busca preservar y } \\
\text { respetar las reservas de } \\
\text { la naturaleza. }\end{array}$ & $\begin{array}{l}\text { Duisburg Nord } \\
\text { Landscape Park } \\
\text { (Alemania) }\end{array}$ & $\begin{array}{l}\text { Ambiental } \\
\text { Física }\end{array}$ & Medioambiental & $\begin{array}{l}\text { Fondos públicos } \\
\text { y privados }\end{array}$ \\
\hline \multirow{4}{*}{$\begin{array}{l}\text { Protección } \\
\text { Patrimonial }\end{array}$} & \multirow{4}{*}{$\begin{array}{l}\text { Su objetivo } \\
\text { primordial se basa } \\
\text { en la significancia } \\
\text { del patrimonio, } \\
\text { custodiando su } \\
\text { importancia histórica } \\
\text { y cultural para la } \\
\text { ciudad, además de la } \\
\text { protección de la fauna y } \\
\text { flora donde se garantiza } \\
\text { subsistencia de los } \\
\text { seres vivos, evitando } \\
\text { la contaminación y } \\
\text { la depredación de } \\
\text { recursos. }\end{array}$} & $\begin{array}{l}\text { Antigua Plaza de } \\
\text { Mercado, Tunja } \\
\text { (Colombia) }\end{array}$ & Física & Económico & $\begin{array}{l}\text { Asociaciones } \\
\text { público-privadas }\end{array}$ \\
\hline & & $\begin{array}{l}\text { Distillery District, } \\
\text { Toronto (Canadá) }\end{array}$ & Física & Económico & Fondos privados \\
\hline & & $\begin{array}{l}\text { High Line Elevated } \\
\text { Park, Nueva York } \\
\text { (Estados Unidos de } \\
\text { América) }\end{array}$ & Física & Medioambiental & Donaciones \\
\hline & & $\begin{array}{l}\text { Duisburg Nord } \\
\text { Landscape Park } \\
\text { (Alemania) }\end{array}$ & Física & Medioambiental & $\begin{array}{l}\text { Fondos públicos } \\
\text { y privados }\end{array}$ \\
\hline \multirow{3}{*}{ Consolidación } & $\begin{array}{l}\text { Debido al crecimiento } \\
\text { urbano, el cual repre- } \\
\text { senta la utilización de } \\
\text { grandes extensiones } \\
\text { de suelo para satisfacer }\end{array}$ & $\begin{array}{l}\text { Tranvía de } \\
\text { Ayacucho, } \\
\text { Medellín } \\
\text { (Colombia) }\end{array}$ & Espacial & Económico & Fondos públicos \\
\hline & $\begin{array}{l}\text { las necesidades que } \\
\text { se presentan, se hace } \\
\text { necesaria la adaptación } \\
\text { de espacios conforme } \\
\text { al crecimiento de la }\end{array}$ & $\begin{array}{l}\text { Avenida Jiménez, } \\
\text { Bogotá D.C. } \\
\text { (Colombia) }\end{array}$ & $\begin{array}{l}\text { Social } \\
\text { Ambiental }\end{array}$ & Social & Fondos públicos \\
\hline & $\begin{array}{l}\text { ciudad, donde su repre- } \\
\text { sentación se basa en la } \\
\text { sostenibilidad. }\end{array}$ & $\begin{array}{l}\text { Área Urbana } \\
\text { Curitiba (Brasil) }\end{array}$ & Ambiental & Social & $\begin{array}{l}\text { Fondos públicos } \\
\text { y privados }\end{array}$ \\
\hline
\end{tabular}




\begin{tabular}{|c|c|c|c|c|c|}
\hline Tipo & Descripción & $\begin{array}{c}\text { Casos } \\
\text { Representativos } \\
\text { Ciudad y país }\end{array}$ & Intervención & $\begin{array}{l}\text { Sistema de } \\
\text { Ciudad }\end{array}$ & $\begin{array}{c}\text { Modelo de } \\
\text { Financiamiento } \\
\text { Identificado }\end{array}$ \\
\hline \multirow{3}{*}{$\begin{array}{l}\text { Renovación } \\
\text { Urbana }\end{array}$} & \multirow{3}{*}{$\begin{array}{l}\text { Toda intervención que } \\
\text { se hace sobre una zona } \\
\text { que bien esté deterio- } \\
\text { rada o abandonada, } \\
\text { con el fin de rehabilitar } \\
\text { el espacio para prestar } \\
\text { el mismo servicio que } \\
\text { ofrecía o darle un uso } \\
\text { totalmente diferente al } \\
\text { inicial. }\end{array}$} & $\begin{array}{l}\text { Duisburg Nord } \\
\text { Landscape Park } \\
\text { (Alemania) }\end{array}$ & $\begin{array}{l}\text { Física } \\
\text { Ambiental }\end{array}$ & Medioambiental & $\begin{array}{l}\text { Fondos públicos } \\
\text { y privados }\end{array}$ \\
\hline & & $\begin{array}{l}\text { Tianjin Qiaoyuan } \\
\text { Wetland Park } \\
\text { (China) }\end{array}$ & Ambiental & Medioambiental & Fondos públicos \\
\hline & & $\begin{array}{l}\text { Río } \\
\text { Cheonggyecheon, } \\
\text { Seúl (Corea del Sur) }\end{array}$ & Ambiental & $\begin{array}{l}\text { Medioambiental } \\
\text { y Físico }\end{array}$ & Fondos públicos \\
\hline Desarrollo & $\begin{array}{l}\text { Transformación } \\
\text { encaminada a la inno- } \\
\text { vación por medio de la } \\
\text { aplicación de nuevas } \\
\text { tecnologías con el fin de } \\
\text { configurar una sociedad } \\
\text { más actual, con } \\
\text { mayores posibilidades } \\
\text { de desarrollo a partir } \\
\text { de la modernización y } \\
\text { el aprovechamiento de } \\
\text { espacios buscando el } \\
\text { beneficio de la gente. }\end{array}$ & $\begin{array}{l}\text { Pabellón de Pallets, } \\
\text { Christchurch } \\
\text { (Nueva Zelanda) }\end{array}$ & Social & Social & Donaciones \\
\hline
\end{tabular}

Tabla. Propuesta tipológica de transformaciones urbanas. Fuente: elaboración propia

\section{Discusión: prospectiva de transformaciones urbanas en las ciudades de Colombia}

Según investigaciones disponibles en la página oficial de la Organización de Naciones Unidas (ONU), junto con diferentes estudios realizados para evaluar la situación mundial de la humanidad y recursos naturales, se destaca la sobrepoblación que ha surgido en los últimos años; se estima que para el año 2030 la tierra tendrá una población de 8.500 millones de personas. Además de ello se afirma que la escasez de agua afecta a cuatro de cada 10 personas y en cuanto al cambio climático, en el periodo comprendido entre 1880 y 2012 los océanos se han calentado, las cantidades de nieve y hielo han disminuido y el nivel del mar ha subido. Con ello y otras complicaciones que se han desarrollado con el transcurso de los años, se advierte la necesidad de actuar ante esta problemática, estableciendo estrategias que logren mitigar los impactos negativos a la salud y al medio ambiente. En este caso una de las medidas eficientes para llevar a cabo esta tarea se basa en el papel que cumplen las transformaciones urbanas en la planeación y crecimiento de las ciudades, basándose en la reestructuración territorial, buscando integrar $\mathrm{u}$ homogeneizar la sociedad, donde el crecimiento de la ciudad no se vea sectorizado por el fenómeno del capitalismo, sino que más bien se logre una distribución temporo-espacial uniforme, en la cual a partir de ello sea posible generar sostenibilidad, desarrollando nuevas actividades económicas que beneficien a la mayoría; con ello se espera lograr erradicar la desigualdad social que se presenta en gran parte de los países latinoamericanos.

En los casos de las transformaciones urbanas expuestos anteriormente y otros casos que han 
resultado exitosos en diferentes países, se evidencia el impacto ambiental, social, cultural y económico que aquellas generan en el entorno urbano; se distinguen las nuevas técnicas de manejo de vegetación en infraestructuras y espacios verdes en la ciudad, el mantenimiento de construcciones significativas para el lugar, la conservación del patrimonio y con ello, el arte, el ocio y las actividades económicas que se desarrollan en torno a esta nueva modalidad de regeneración de espacios públicos, además de ser una herramienta que garantiza lugares más seguros y limpios. En el contexto colombiano se aprecian diversos escenarios de ejecución de proyectos ligados a las transformaciones urbanas, los cuales promueven el progreso y desarrollo del país; a raíz de ello es necesario el aprovechamiento de estos espacios que componen la ciudad, creando ciudades sostenibles, en términos de conservación del medio ambiente, preservación del patrimonio cultural, movilidad urbana, infraestructura e innovación; se toma como referencia la adecuación del Metro de Medellín, siendo uno de los ejemplos más representativos de transformación urbana, reflejando la globalización que surge en torno a dicho proyecto como el crecimiento de la ciudad, el auge del turismo, el crecimiento económico, la creación de nuevos mercados y empleos, y la cultura, entre otros.

En Colombia es de vital importancia la participación de actores que permitan el desarrollo de las transformaciones urbanas; en el contexto económico la mayoría de los casos implicarán la financiación por empresas del Estado, organizaciones internacionales, asociaciones público privadas, bancos, entre otros organismos que son capaces de cubrir gastos asociados a los proyectos que sean planteados; dependiendo la intervención que se vaya a realizar también deberá participar la comunidad en general, ellos son las personas involucradas directamente con el proyecto, de ellos depende el éxito o decadencia del mismo, la cultura ciudadana debe ir orientada al sentido de pertenencia que se tiene con la ciudad y para la ciudad. En cuanto a la propuesta tipológica -considerada factible en el contexto colombiano-, se propone entender una transformación urbana, no sólo como una intervención en la que se rehabilitan lugares que no cumplen con una actividad productiva en la sociedad, sino también como la reingeniería basada en nuevas estrategias para convertir las ciudades en ciudades más inclusivas, con nuevas formas de vida sostenibles, la gestión del ambiente y los recursos naturales urbanos, la movilidad, la seguridad, la recreación, el diario vivir, las actividades económicas, la cultura ciudadana, la preservación de la arquitectura y el patrimonio, entre muchos otros, en un solo entorno. Estos elementos ayudarán a combatir las consecuencias negativas ocasionadas por el crecimiento del sector urbano y que están afectando la calidad de vida de los ciudadanos.

Finalmente, es primordial recalcar el papel que desempeña la renovación urbana en el cumplimento de los objetivos para la creación de un desarrollo sostenible, establecido por el Programa de las Naciones Unidas para el Desarrollo (PNUD); dentro de sus diecisiete objetivos se encuentra el de la construcción de ciudades y comunidades sostenibles, lo cual sugiere que a partir del aumento de la población, lograrlo implica la transformación en la construcción y administración de los espacios públicos (ONU, 2015).

\section{Conclusiones}

Es acertado señalar que la transformación urbana desarrolla de manera fundamental acciones orientadas a recuperar lugares y espacios abandonados o sin un uso específico que representan molestias en su etapa de desuso; por tanto, su habilitación permite la prestación de servicios urbano ambientales, al mismo tiempo que fomentan actividades sociales y económicas o cualquier otra actividad que aporte al buen funcionamiento de la ciudad; lo anterior se traduce en claros beneficios en las dimensiones ambiental, social y económica en área urbanas y periféricas. En este sentido, si bien la clasificación tipológica propuesta comprende clases orientadas al sistema físico, el sistema medioambiental, el sistema social y el sistema 
económico, debe indicarse que es imposible no mezclarlos; es importante destacar que en la ejemplificación de cada tipo de transformación se tuvo en cuenta la característica con más participación o un aporte de mayor significancia en cada sistema; no obstante lo anterior, la mayoría de las transformaciones urbanas estudiadas presentan beneficios en el conjunto de sistemas y dimensiones de la sostenibilidad urbana.

Es necesario contar con una tipología de transformaciones urbanas que permita distinguir clases o familias con base en los innumerables casos que se han logrado llevar a cabo y han concluido exitosamente; se requiere profundizar en la conceptualización de los tipos de transformaciones para ser incluidas en los planes de ordenamiento territorial de las ciudades y así facilitar el trabajo de planeación y gestión de las ciudades a los administradores urbanos. La tipología proyectada en cada ciudad, región o territorio también debe ajustarse y encontrarse en consonancia con los parámetros y lineamientos establecidos por cada gobierno en sus documentos oficiales que definen la política de desarrollo y de expansión urbana. En todo caso, sin distinción del tipo de transformación, para ser considerada bajo dicha denominación, el objetivo de ésta debe encaminarse hacia la mejora de la calidad de vida urbana a través de los servicios que ofrece.

La implementación de las transformaciones urbanas en las ciudades del mundo muestra resultados excelentes; se ha generado un incremento de las actividades que se desarrollan en torno a las áreas recuperadas y renovadas, y de allí una mejora significativa de la calidad de vida de los ciudadanos, la apropiación e identidad urbana, la protección del ambiente urbano, además de una potenciación de la economía y del interés en efectuar la renovación de los lugares y espacios perdidos y desaprovechados de las ciudades. Como respuesta a las problemáticas ambientales urbanas, en el contexto global y latinoamericano se plantean proyectos y modelos de transformación urbana que han sido efectivos y se consideran oportunos para llevar a cabo en
Colombia; resultará importante contemplar en el corto plazo incorporar dentro de las políticas públicas urbanas, regionales y territoriales de desarrollo, aquellos relacionados con infraestructuras públicas como parques, ejes ambientales, de gran superficie y lineales, que garanticen la preservación y adecuada gestión de los recursos ambientales urbanos. Dichos proyectos de transformación urbana, que deben ser introducidos en la política nacional de desarrollo, deben constituirse en elementos orientados a la sostenibilidad en las dimensiones ambiental, social y económica tomando como derroteros proporcionar zonas urbanas ambientalmente limpias y saludables, la generación de apropiación e identidad urbana, la atracción del turismo, el desarrollo comercial y económico. Para las ciudades intermedias, incluyendo las de Colombia, se identifican como de especial interés las transformaciones urbanas relativas al desarrollo de sistemas de transporte, que permitan satisfacer las necesidades de movilidad de los usuarios de manera accesible y sostenible, y contribuyan a la reducción de las externalidades negativas del transporte urbano.

\section{Referencias bibliográficas}

ASLA (2010). Tianjin Qiaoyuan Park: the adaptation palettes. American Society of Landscape Architects. Recuperado de: [https://www.asla. org/2010awards/033.html].

Bueno, J. (2016). Tranvía de Medellín, primer sistema de tranvía moderno de Latinoamérica. ROP, 3572, pp. 98-106. Recuperado de: [http:// ropdigital.ciccp.es/pdf/publico/2016/2016_ enero_3572_09.pdf].

Ciudades del Futuro (2012, 3 de agosto). Reutilizando estructuras: High line elevated park [Entrada de blog]. Recuperado de: [https:// ciudadesdelfuturo.es/reutilizando-estructurashigh-line-elevated-park.php].

Icecomex (2007). Pallets. [Entrada de blog]. Consultorio de Comercio Exterior de la Univer- 
sidad Icesi. Recuperado de: [https://www.icesi. edu.co/blogs/icecomex/2007/04/27/pallets/].

Culture Trip (24 de octubre de 2016). The History of The Distillery District in 1 Minute. Recuperado de: [https://theculturetrip.com/north-america/ canada/articles/the-history-of-the-distillerydistrict-in-1-minute/].

Development Asia (13 de mayo de 2016). Case study: revitalizing a city by reviving a stream. Asia Development Bank. Recuperado de: [https://development.asia/case-study/ revitalizing-city-reviving-stream].

Diputación de Barcelona (2008). Ciudades en (re) construcción: necesidades sociales, transformación y mejora de barrios. Colección de Estudios, Serie Territorio 5. Barcelona: Diputación de Barcelona.

Donat, B. (5 de diciembre de 2015). Distillery District, de destilería de whisky a barrio de diseño. EL TIEMPO, Toronto. Recuperado de: https://www.eltiempo.com/archivo/documen to/CMS-16450019

EPM (28 de marzo de 2014). EPM - Camino al Barrio -Historia Tranvía Antiguo. Empresas Públicas de Medellín. [Archivo de video]. Recuperado de: [https://www.youtube.com/ watch? $\mathrm{v}=\mathrm{cm} 3 \mathrm{z} 5 \mathrm{O} 2 \mathrm{LGfo}$.

Fukuda, I. (2010). Planeación urbana en Curitiba. Quivera, 12(1), pp. 52-69. Recuperado de: [http://www.celadel.org/textos/curitiba/ Planeamiento\%20Urbano.pdf].

Green Planet Architects (2018a). Turenscape. Recuperado de: [https://www.greenplanetarchitects. com/en/architect/landscape/turenscape].

Green Planet Architects (2018b). Tianjin Qiaoyuan Wetland Park. Recuperado de: [https:// www.greenplanetarchitects.com/en/project/ landscape/tianjin-qiaoyuan-wetland-park].

Hammond, R. (2011). Construyendo un parque en el cielo. [Archivo de video]. TED Ideas worth spreading. Recuperado de: [https://www.ted. com/talks/robert_hammond_building_a_ park_in_the_sky?language $=\mathrm{es}]$.
Higueras, E. (2013). La ciudad como ecosistema urbano. Monografía (artículo de discusión). Madrid: Universidad Politécnica de Madrid. Recuperado de: [http://oa.upm.es/16625/1/ Ecosistema.pdf].

Kontentreal LLC. (2008). Seoul: The Stream of Consciousness [Archivo de vídeo]. Recuperado de: [https://www.youtube.com/watch? $\mathrm{v}=\mathrm{o}-\mathrm{kW}$ WIVVib0].

Landschaftspark Duisburg-Nord (s.f.). Denkmal Hüttenwerk. Recuperado de: [https://www. landschaftspark.de/rundweg-industriegeschichte/denkmal-huttenwerk/].

Latz und Partner (s.f.). Postindustrial Landscapes. Recuperado de: [https://www.latzundpartner. de/en/projekte/postindustrielle-landschaften/].

Linde, P. (2017). ¿Qué tiene el transporte público de Medellín para ser un modelo en Latinoamérica? El País. Recuperado de: [https://elpais.com/elpais/2017/03/01/plane ta_futuro/1488327644_918944.html].

Lindner, C. y Rosa, B. (2016). Deconstructing the High Line. Postindustrial Urbanism and the Rise of the Elevated Park. New Brunswick: Rutgers University Press Recuperado de: [https://www.researchgate.net/ publication/321980523_Deconstructing_the_ high_line_Postindustrial_Urbanism_and_the_ rise_of_the_Elevated_Park].

Lozano, L. H. (2016). Caso Eje Ambiental: urbanismo táctico en Bogotá, Colombia. ResearchGate. DOI: [10.13140/RG.2.1.3763.6084].

Márquez, J.W. (2012). El tranvía eléctrico de Medellín (Colombia) y su aporte al proceso de modernización urbana: 1920-1951. Historelo Revista de Historia Regional y Local, 4(7), pp. 131-155. Recuperado de: [http://www.scielo. org.co/pdf/histo/v4n7/v4n7a06.pdf].

Martínez, C. (2013). "Pabellón de Pallets": Ciudadanos logran regenerar un nuevo espacio público en Nueva Zelanda. Plataforma urbana. Recuperado de: [http://www.plataformaurbana. cl/archive/2013/09/29/pabellon-de-pallets- 
-ciudadanos-logran-regenerar-un-nuevo-espacio-publico-en-nueva-zelanda/].

Marván, A. (16 de abril de 2018). Toronto: una historia empapada en whisky. Avianca en revista. Recuperado de: [https://www.aviancaenrevista. com/experiencias/toronto-historia-whisky/].

Metro de Medellín (2015). El tranvía y sus dos cables, un proyecto regional. Revista Metro. Recuperado de: [https://www.metrodemedellin.gov.co/Portals/1/Images/Contenido/ REVISTAS-OTROS/2015-Revista-NuestroTranvia-15-09-2015.pdf].

Metro de Medellín (11 de abril de 2016). El metro se reafirma como un sistema amigable con el medio ambiente. Recuperado de: [https:// www.metrodemedellin.gov.co/al-d\%C3\%ADa/ noticias-metro/artmid/6905/articleid/26/ el-metro-se-reafirma-como-un-sistemaamigable-con-el-medio-ambiente].

Mishra, S.A. y Pandit, R.K. (2013). Urban transformation and role of architecture towards social sustainability. International Journal of Engineering Research and Development, 5(7), pp. 16-20.

Motoa, F. (30 de agosto de 2018). La avenida Jiménez cumple 80 años de haber sido inaugurada. Bogotá: El Tiempo. Recuperado de: [https:// www.eltiempo.com/bogota/asi-era-antes-laavenida-jimenez-que-cumple-80-anos-262002].

ONU (2015). Objetivos de Desarrollo Sostenible. Organización de Naciones Unidas. Recuperado de: [https://www.un.org/sustainabledevelo pment/es/objetivos-de-desarrollo-sostenible/].

Paisajismo Digital (10 de octubre de 2017). Curitiba, modelo de urbanismo verde y sustentable. Recuperado de: [https://paisajismodigital.com/ blog/curitiba-urbanismo-verde-y-sustentable/].
Pascuali, M. (2020). Ciudades más pobladas de Brasil en 2020. Statista. Recuperado de: [https://es.statista.com/estadisticas/599077/ ciudades-mas-grandes-en-brasil-en/].

Quintero, J. R. y Quintero, L. E. (2018). Estrategias de transformación urbana en la ciudad de Tunja, Colombia: infraestructuras públicas $y$ recursos naturales en la Comuna 5 Centro. Medellín, Colombia: Institución Universitaria Colegio Mayor de Antioquia

Rottle, N. y Lacson, D. (2011). Tianjin Qiaoyuan Park: The Adaptation Palettes. Landscape Performance Series. Landscape Architecture Foundation. Recuperado de: [https://landscapeperformance.org/case-study-briefs / tianjin-qiaoyuan-park-the-adaptation-palettes].

Sánchez, N. (25 de octubre de 2017). High Line: un parque levantado sobre rieles en New York. El caribe. Recuperado de: [https://m.elcaribe.com. do/2017/10/25/high-line-un-parque-levantadosobre-rieles-en-new-york/].

Stilgenbauer, J. (2005). Landschaftspark DuisburgNord - Duisburg, Germany [2005 EDRA/Places Award - Design]. Places, 17(3), pp. 6-9. Recuperado de: [https://placesjournal.org/assets/ legacy/pdfs/landschaftspark-duisburg-nord. pdf].

Velásquez, C.J. (2012). Ciudad y desarrollo sostenible. Primera Edición. Barranquilla-Bogotá D.C.: Editorial Universidad del Norte, ECOE Ediciones.

Yu, K. (2013). Proyectar el paisaje como un ecosistema vivo. Paisea (24), pp. 104-111 Recuperado de: [http://www.paisea.com/wpcontent/uploads/PAISEA24.-ART-5.pdf].

Zeren, N. y Gürler, E. (2011). Conceptual challenges on urban transformation. AZ ITU Journal of the Faculty of Architecture, 8(1), pp. 10-24. 